\title{
Optimality conditions for strict minimizers of higher-order in semi-infinite multi-objective optimization
}

\section{Guolin Yu*}

\section{"Correspondence:} guolin_yu@126.com Institute of Applied Mathematics, Beifang University of Nationalities, Yinchuan, Ningxia 750021, P.R. China

\begin{abstract}
This paper is devoted to the study of optimality conditions for strict minimizers of higher-order for a non-smooth semi-infinite multi-objective optimization problem. We propose a generalized Guignard constraint qualification and a generalized Abadie constraint qualification for this problem under which necessary optimality conditions are proved. Under the assumptions of generalized higher-order strong convexity for the functions appearing in the formulation of the non-smooth semi-infinite multi-objective optimization problem, three sufficient optimality conditions are derived.
\end{abstract}

MSC: Primary 90C34; 90C40; secondary 49J52

Keywords: optimality conditions; multi-objective optimization; semi-infinite optimization; strict minimizer of higher-order

\section{Introduction}

In recent years, there has been considerable interest in the so-called semi-infinite multiobjective optimization problems (SIMOPs), which is the simultaneous minimization of finitely many scalar objective functions subject to an infinitely many constraints. SIMOPs have been investigated intensively by many researchers from several different perspectives. For example, the pseudo-Lipschitz property and the semicontinuity of the efficient solution map under some types of perturbation with respect to a parameter have been discussed in [1-5]. The density of the set of all stable convex semi-infinite vector optimization problems has been established in [6]. However, the work on optimality conditions for SIMOPs is limited. Here we should mention that the authors in [7] have examined the optimality conditions and duality relations in SIMOPs involving differentiable functions, whose constraints are required to depend continuously on an index $t$ belonging to a compact set $T$. For non-smooth semi-infinite multi-objective optimization problems, work has been done to obtain necessary optimality conditions for weakly efficient solutions and sufficient optimality conditions for efficient solutions by presenting several kinds of constraint qualifications and imposing assumptions of generalized convexity (see [8]), and to establish necessary and sufficient conditions for (weakly) efficient solutions of SIMOPs by applying some advanced tools of variational analysis and generalized differentiation and

(c) $2016 \mathrm{Yu}$. This article is distributed under the terms of the Creative Commons Attribution 4.0 International License (http://creativecommons.org/licenses/by/4.0/), which permits unrestricted use, distribution, and reproduction in any medium, provided you give appropriate credit to the original author(s) and the source, provide a link to the Creative Commons license, and indicate if changes were made. 
proposing the concepts of (strictly) generalized convex functions defined by using the limiting subdifferential of locally Lipschitz functions (see [9]). It is worth noticing that all of the above mentioned literature studies only weakly efficient solutions or efficient solutions of SIMOPs.

On the other hand, a continuing interest in the theory of multi-objective optimization is to define and characterize its solutions. Besides the weak efficiency and efficiency mentioned above, a meaningful solution concept called a strict efficient solution of higherorder (also called a strict minimizer of higher-order) was recently extended by Jiménez in [10] from the strict minimizer of higher-order in scalar optimization given by Auslender in [11] and Ward in [12]. Recently, Bhatia [13] established necessary and sufficient optimality conditions for strict efficiency of higher-order in multi-objective optimization under the basic regularity condition and generalized higher-order strong convexity assumption, respectively.

In this paper, we introduce the notion of a semi-strict minimizer of higher-order for a semi-infinite multi-objective optimization problem, which includes arbitrary many (possibly infinite) inequality constraints. For the purpose of investigating this new solution concept, we found that the notion of convexity that appears to be most appropriate in the development of sufficient optimality conditions is the strong convexity of higher-order [14].

The rest of this paper is organized as follows. In Section 2, some basic notations and results of non-smooth and convex analysis are reviewed, and the concept of a semistrict minimizer for a semi-infinite multi-objective optimization problem is presented. In Section 3, we introduce the generalized Guignard constraint qualification and Abadie constraint qualification for SIMOPs. Necessary optimality conditions of Karush-KuhnTuchker type are derived under these two constraint qualifications. Finally, in Section 4, three sufficient optimality conditions for SIMOPs are obtained under the assumption of some generalized strong convexity of higher-order.

\section{Notations and preliminaries}

Throughout the paper, we let $\mathbb{R}^{n}$ be the $n$-dimensional Euclidean space endowed with the Euclidean norm $\|\cdot\|, X$ be a convex subset of $\mathbb{R}^{n}$, and $m \geq 1$ be a positive integer. Let $W$ be a subset of $\mathbb{R}^{n}$. We use cl $W$, co $W$, and cone $W$ to denote the closure of $W$, the convex hull of $W$, and the conic hull of $W$ (i.e., the smallest convex cone containing $W$ ), respectively.

Definition 2.1 (see [15-17]) Let $W$ be a nonempty subset of $\mathbb{R}^{n}$. The tangent cone to $W$ at $\bar{x} \in \mathrm{cl} W$ is the set defined by

$$
\begin{aligned}
T(W ; \bar{x}):= & \left\{h \in \mathbb{R}^{n}: h=\lim _{n \rightarrow \infty} t_{n}\left(x^{n}-\bar{x}\right) \text { such that } x^{n} \in W,\right. \\
& \left.\lim _{n \rightarrow \infty} x^{n}=\bar{x} \text { and } t_{n}>0 \text { for all } n=1,2, \ldots\right\} .
\end{aligned}
$$

Recall that a function $\varphi: X \rightarrow \mathbb{R}$ is Lipschitz at $\bar{x} \in X$ if there exists a positive constant $K$ such that

$$
|\varphi(x)-\varphi(\bar{x})| \leq K\|x-\bar{x}\| \quad \text { for all } x \in X
$$

where $K$ is called the rank of $\varphi$ at $\bar{x}$. $\varphi$ is said to be Lipschitz on $X$ if $\varphi$ is Lipschitz at each $x \in X$. Suppose that $\varphi$ is Lipschitz at $\bar{x} \in X$, then Clarke's generalized directional derivative 
of $\varphi$ at $\bar{x} \in X$ in the direction $v \in \mathbb{R}^{n}$, denoted by $\varphi^{0}(\bar{x}, v)$, is defined as

$$
\varphi^{0}(\bar{x}, v)=\limsup _{(x, t) \rightarrow\left(\bar{x}, 0^{+}\right)} \frac{\varphi(x+t v)-\varphi(x)}{t} .
$$

Clarke's generalized gradient of $\varphi$ at $\bar{x} \in X$, denoted by $\partial \varphi(\bar{x})$, is defined as

$$
\partial \varphi(\bar{x})=\left\{\xi \in \mathbb{R}^{n}: \varphi^{0}(\bar{x}, v) \geq\langle\xi, v\rangle \text { for all } v \in \mathbb{R}^{n}\right\} .
$$

It is well known that $\partial \varphi(\bar{x})$ is a nonempty convex compact set in $\mathbb{R}^{n}$.

Definition 2.2 (see [18]) Let $\varphi: \mathbb{R}^{n} \rightarrow \mathbb{R}$ be Lipschitz at $\bar{x} \in \mathbb{R}^{n}$. It is said that $\varphi$ admits a strict derivative at $\bar{x}$, an element of $\mathbb{R}^{n}$, denoted by $D_{s} \varphi(\bar{x})$, provided that, for each $x \in \mathbb{R}^{n}$, the following holds:

$$
\lim _{\left(x^{\prime}, t\right) \rightarrow(\bar{x}, 0)} \frac{\varphi\left(x^{\prime}+t x\right)-\varphi\left(x^{\prime}\right)}{t}=\left\langle D_{s} \varphi(\bar{x}), x\right\rangle
$$

If $\varphi$ admits a strict derivative at $\bar{x}$, then $\varphi$ is called strictly differentiable at $\bar{x}$.

Lemma 2.1 (see [19]) Let $\varphi, \varphi_{1}$, and $\varphi_{2}$ be Lipschitz from $X$ to $\mathbb{R}$, and $\bar{x} \in X$. Then the following properties hold:

(a) $\varphi^{0}(\bar{x}, v)=\max \{\langle\xi, v\rangle: \xi \in \partial \varphi(\bar{x})\}$, for all $v \in \mathbb{R}^{n}$.

(b) $\partial(\lambda \varphi(\bar{x}))=\lambda \partial \varphi(\bar{x})$, for all $\lambda \in \mathbb{R}$.

(c) $\partial\left(\varphi_{1}+\varphi_{2}\right)(\bar{x}) \subset \partial \varphi_{1}(\bar{x})+\partial \varphi_{2}(\bar{x})$.

Now, we recall the definition of the strong convexity of order $m$ for a Lipschitz function.

Definition 2.3 (see $[13,14]$ ) Let $\varphi: X \rightarrow \mathbb{R}$ be Lipschitz at $\bar{x} \in X$.

(a) $\varphi$ is said to be strongly convex of order $m$ at $\bar{x}$ if there exists a constant $c>0$ such that for each $x \in X$ and $\xi \in \partial \varphi(\bar{x})$

$$
\varphi(x)-\varphi(\bar{x}) \geq\langle\xi, x-\bar{x}\rangle+c\|x-\bar{x}\|^{m} .
$$

(b) $\varphi$ is said to be strongly quasiconvex of order $m$ at $\bar{x}$ if there exists a constant $c>0$ such that, for each $x \in X$ and $\xi \in \partial \varphi(\bar{x})$,

$$
\varphi(x) \leq \varphi(\bar{x}) \quad \Rightarrow \quad\langle\xi, x-\bar{x}\rangle+c\|x-\bar{x}\|^{m} \leq 0 .
$$

Based upon the above definition of a strongly convex function of order $m$, we define the following generalized strong convexities of order $m$ for a Lipschitz function.

Definition 2.4 Let $\varphi: X \rightarrow \mathbb{R}$ be Lipschitz at $\bar{x} \in X$.

(a) $\varphi$ is strictly strong convex of order $m$ at $\bar{x}$ if there exists a constant $c>0$ such that, for each $x \in X$ with $x \neq \bar{x}$ and $\xi \in \partial \varphi(\bar{x})$,

$$
\varphi(x)-\varphi(\bar{x})>\langle\xi, x-\bar{x}\rangle+c\|x-\bar{x}\|^{m} .
$$


(b) $\varphi$ is strictly strong quasiconvex of order $m$ at $\bar{x}$ if there exists a constant $c>0$ such that, for each $x \in X$ with $x \neq \bar{x}$ and any $\xi \in \partial \varphi(\bar{x})$,

$$
\varphi(x) \leq \varphi(\bar{x}) \quad \Rightarrow \quad\langle\xi, x-\bar{x}\rangle+c\|x-\bar{x}\|^{m}<0 .
$$

The next Lemma gives a basic property of generalized higher-order strong convexities, which will be used in Section 4.

Proposition 2.1 Let $\varphi_{i}: X \rightarrow \mathbb{R}$ be Lipschitz at $\bar{x} \in X, i=0,1,2, \ldots, s$. Suppose that $\varphi_{0}$ is a strictly strong convex function of order $m$ and $\varphi_{1}, \varphi_{2}, \ldots, \varphi_{s}$ are strongly convex functions of order $m$ at $\bar{x}$. If $\lambda_{0}>0$ and $\lambda_{i} \geq 0$ for $i=1,2, \ldots, s$, then $\sum_{i=0}^{s} \lambda_{i} \varphi_{i}$ is strictly strong convex of order $m$ at $\bar{x}$.

Proof It is evident that the function $\sum_{i=0}^{s} \lambda_{i} \varphi_{i}$ is Lipschitz at $\bar{x}$. Thus, we get

$$
\partial\left(\sum_{i=0}^{s} \lambda_{i} \varphi_{i}\right)(\bar{x}) \neq \emptyset
$$

Taking $\xi \in \partial\left(\sum_{i=0}^{s} \lambda_{i} \varphi_{i}\right)(\bar{x})$. It follows from Lemma 2.1 that

$$
\partial\left(\sum_{i=0}^{s} \lambda_{i} \varphi_{i}\right)(\bar{x}) \subset \sum_{i=0}^{s} \partial\left(\lambda_{i} \varphi_{i}\right)(\bar{x})=\sum_{i=0}^{s} \lambda_{i} \partial \varphi_{i}(\bar{x}) .
$$

This means that there exist $\xi_{i} \in \partial \varphi_{i}(\bar{x}), i=0,1, \ldots, s$, such that

$$
\xi=\sum_{i=0}^{s} \lambda_{i} \xi_{i}
$$

Since $\varphi_{0}$ is strictly strong convex of order $m$ at $\bar{x}$ and $\varphi_{i}, i=1,2, \ldots, s$, is strongly convex of order $m$ at $\bar{x}$, we derive that there exist $c_{i}>0, i=0,1, \ldots, s$, such that, for all $x \in \mathbb{R}^{n}$,

$$
\left\{\begin{array}{l}
\varphi_{0}(x)-\varphi_{0}(\bar{x})>\left\langle\xi_{0}, x-\bar{x}\right\rangle+c_{0}\|x-\bar{x}\|^{m}, \\
\varphi_{i}(x)-\varphi_{i}(\bar{x}) \geq\left\langle\xi_{i}, x-\bar{x}\right\rangle+c_{i}\|x-\bar{x}\|^{m} \quad \text { for all } i=1,2, \ldots, s
\end{array}\right.
$$

which implies that

$$
\sum_{i=0}^{s} \lambda_{i}\left(\varphi_{i}(x)-\varphi_{i}(\bar{x})\right)>\sum_{i=0}^{s} \lambda_{i}\left\langle\xi_{i}, x-\bar{x}\right\rangle+\left(\sum_{i=0}^{s} \lambda_{i} c_{i}\right)\|x-\bar{x}\|^{m}
$$

Therefore, we get

$$
\left(\sum_{i=0}^{s} \lambda_{i} \varphi_{i}\right)(x)-\left(\sum_{i=0}^{s} \lambda_{i} \varphi_{i}\right)(\bar{x})>\langle\xi, x-\bar{x}\rangle+c\|x-\bar{x}\|^{m},
$$

where $c=\sum_{i=0}^{s} \lambda_{i} c_{i}$. This completes the proof of the proposition. 
Consider the following semi-infinite multi-objective optimization problem:

(P) Minimize $f(x)=\left(f_{1}(x), f_{2}(x), \ldots, f_{p}(x)\right)$

subject to $g_{t}(x) \leq 0 \quad$ for $t \in T$,

$x \in X$,

where $f_{i}, i \in P=\{1,2, \ldots, p\}$, and $g_{t}, t \in T$ are Lipschitz from $X$ to $\mathbb{R}$, and the index set $T$ is arbitrary, not necessarily finite (but nonempty). The feasible set of $(\mathrm{P})$ is denoted by $\Omega$,

$$
\Omega:=\left\{x \in X: g_{t}(x) \leq 0, \forall t \in T\right\} .
$$

For a given $\bar{x} \in \Omega$, set

$$
\hat{T}(\bar{x}):=\left\{t \in T: g_{t}(\bar{x})=0\right\} .
$$

In the sequel, we use the following notations. For $x, y \in \mathbb{R}^{n}$.

(i) $f(x)<f(y) \Leftrightarrow f_{i}(x)<f_{i}(y)$ for every $i \in P$;

(ii) $f(x) \nless f(y)$ is the negation of $f(x)<f(y)$;

(iii) $f(x) \leq f(y) \Leftrightarrow f_{i}(x) \leq f_{i}(y)$ for every $i \in P$, but there is at least one $i_{0} \in P$ such that $f_{i_{0}}(x)<f_{i_{0}}(y)$;

(iv) $f(x) \not \leq f(y)$ is the negation of $f(x) \leq f(y)$.

Definition 2.5 (see [10]) A point $\bar{x} \in \Omega$ is said to be a strict minimizer of order $m$ for (P) if there exists $c=\left(c_{1}, c_{2}, \ldots, c_{p}\right) \in \mathbb{R}^{p}$ with $c_{i}>0, i \in P$, such that

$$
f(x) \nless f(\bar{x})+c\|x-\bar{x}\|^{m} \quad \text { for all } x \in \Omega \text {. }
$$

Definition 2.6 A point $\bar{x} \in \Omega$ is said to be a semi-strict minimizer of order $m$ for (P) if there exists $c=\left(c_{1}, c_{2}, \ldots, c_{p}\right) \in \mathbb{R}^{p}$ with $c_{i}>0, i \in P$, such that

$$
f(x) \not \leq f(\bar{x})+c\|x-\bar{x}\|^{m} \quad \text { for all } x \in \Omega \text {. }
$$

Remark 2.1 It is obvious that if $\bar{x} \in \Omega$ is a semi-strict minimizer of order $m$ for (P), then $\bar{x} \in \Omega$ is a strict minimizer of order $m$ for $(\mathrm{P})$.

Example 2.1 The functions $f_{i}: \mathbb{R} \rightarrow \mathbb{R}, i=1,2$, defined by

$$
f_{1}(x)=\left\{\begin{array}{ll}
x^{4}+e^{x} & \text { if } x \geq 0, \\
x^{4}+1 & \text { if } x<0,
\end{array} \quad f_{2}(x)= \begin{cases}x^{4}+x & \text { if } x \geq 0 \\
x^{4}-x & \text { if } x<0\end{cases}\right.
$$

are Lipschitz at $\bar{x}=0$. It is easy to verify that $\bar{x}$ is a semi-strict minimizer of order 4 with $c=(1,1)$ for the following optimization problem:

$\left(\mathrm{P}_{0}\right) \quad$ Minimize $f(x)=\left(f_{1}(x), f_{2}(x)\right)$ subject to $x \in \mathbb{R}$. 
Motivated by the notion of $a$ linearizing cone at a point to the feasible set of a differentiable multi-objective optimization problem, which was introduced by Maeda in [15], we give the definition of a linearizing cone for the semi-infinite multi-objective optimization problem (P). We first need to define a set.

Let $\bar{x} \in \Omega$ be a semi-strict minimizer of order $m$ for (P), and define

$$
Q^{i}(\bar{x}):=\left\{x \in \mathbb{R}^{n}: f_{k}(x) \leq f_{k}(\bar{x})+c_{k}\|x-\bar{x}\|^{m}, k \in P \text { and } k \neq i\right\} \cap \Omega
$$

It is obvious that $\bar{x} \in Q^{i}(\bar{x}), i \in P$.

Definition 2.7 Let $\bar{x} \in \Omega$. The linearizing cone at $\bar{x}$ is the set defined by

$$
\begin{gathered}
C(\bar{x})=\left\{z \in \mathbb{R}^{n}:\langle\xi, z\rangle \leq 0 \text { for all } \xi \in \partial f_{i}(\bar{x}), i \in P\right. \text { and } \\
\left.\langle\zeta, z\rangle \leq 0 \text { for all } \zeta \in \partial g_{t}(\bar{x}), t \in \hat{T}(\bar{x})\right\} .
\end{gathered}
$$

\section{Necessary conditions}

In this section, we shall examine necessary optimality conditions for a semi-strict (strict) minimizer of order $m$ for $(\mathrm{P})$. we begin with presenting two constraint qualifications, which are the non-smooth, semi-infinite version of the generalized Guignard constraint qualification and generalized Abadie constraint qualification presented in [15] and [16].

Definition 3.1 The problem (P) satisfies the generalized Guignard constraint qualification at a given point $\bar{x} \in \Omega$ which is a semi-strict minimizer of order $m$ for $(\mathrm{P})$ if the following holds: $C(\bar{x}) \subseteq \bigcap_{i=1}^{p} \operatorname{cl}\left[\operatorname{co}\left(T\left(Q^{i} ; \bar{x}\right)\right)\right]$, where $Q^{i}:=Q^{i}(\bar{x})$.

Definition 3.2 The problem (P) satisfies the generalized Abadie constraint qualification at a given point $\bar{x} \in \Omega$ which is a semi-strict minimizer of order $m$ for (P) if the following holds: $C(\bar{x}) \subseteq \bigcap_{i=1}^{p} T\left(Q^{i} ; \bar{x}\right)$.

Next, we recall the generalized Motzkin theorem discussed in [20].

Lemma 3.1 (see [20]) Let $A$ be a compact set in $\mathbb{R}^{n}, B$ an arbitrary set in $\mathbb{R}^{n}$. Suppose that the set cone $B$ is closed. Then either the system

$$
\left\{\begin{array}{l}
\langle a, z\rangle<0 \quad \text { for all } a \in A, \\
\langle b, z\rangle \leq 0 \quad \text { for all } b \in B,
\end{array}\right.
$$

has a solution $z \in \mathbb{R}^{n}$, or there exist integers $\mu$ and $v$, with $0 \leq v \leq n+1$, such that there exist $\mu$ points $a^{i} \in A(i=1,2, \ldots, \mu), v$ points $b^{m} \in B(m=1,2, \ldots, v), \mu$ nonnegative numbers $u_{i}$, with $u_{i}>0$ for at least one $i \in\{1,2, \ldots \mu\}$, and vositive numbers $v_{m}$ for $m \in\{1,2, \ldots \nu\}$, such that

$$
\sum_{i=1}^{\mu} u_{i} a^{i}+\sum_{m=1}^{\nu} v_{m} b^{m}=0,
$$

but never both. 
The following lemma is a generalization of the classical Tucker theorem of the alternative. We use this lemma in the proof of our necessary efficiency result. The proof of the lemma is similar to that of Lemma 3.6 in [17], hence it is omitted.

Lemma 3.2 Let $A^{i} \subset \mathbb{R}^{n}, i \in P$, be compact convex sets, $B$ an arbitrary set in $\mathbb{R}^{n}$. Suppose that, for each $i \in P$, the set $\operatorname{cone}\left(B \cup\left[\bigcup_{j \in P, j \neq i} A^{j}\right]\right)$ is closed. Then either the system

$$
\left\{\begin{array}{l}
\langle a, z\rangle<0 \quad \text { for at least one } i \in P \text { and for all } a \in A^{i} \\
\langle a, z\rangle \leq 0 \text { for all } a \in \bigcup_{i=1}^{p} A^{i} \\
\langle b, z\rangle \leq 0 \quad \text { for all } b \in B
\end{array}\right.
$$

has a solution $z \in \mathbb{R}^{n}$, or there exist $u \in U \equiv\left\{u \in \mathbb{R}^{p}: u>0, \sum_{i=1}^{p} u_{i}=1\right\}, a^{i} \in A^{i}$ for $i \in P$, and integer $v$ with $0 \leq v \leq n+1$, such that there exist $v$ points $b^{m} \in B$, and $v$ positive numbers $v_{m}, m \in\{1,2, \ldots, v\}$, such that

$$
\sum_{i=1}^{p} u_{i} a^{i}+\sum_{m=1}^{v} v_{m} b^{m}=0
$$

but never both.

Lemma 3.3 Let $\bar{x}$ be a semi-strict minimizer of order $m$ for $(\mathrm{P})$, let $f_{i}(x), i \in P$, be Lipschitz at $\bar{x}$ of rank $K_{i}$, for all $t \in T$, let the functions $g_{t}(x)$ be Lipschitz at $\bar{x}$. If the generalized Guignard constraint qualification holds at $\bar{x}$ and $f_{i}(x), i \in P$, are strictly differentiable at $\bar{x}$ or the generalized Abadie constraint qualification holds at $\bar{x}$, then the system

$$
\left\{\begin{array}{l}
\langle\xi, z\rangle<0 \quad \text { for at least one } i \in P \text { and for all } \xi \in \partial f_{i}(\bar{x}) \\
\langle\xi, z\rangle \leq 0 \text { for all } \xi \in \bigcup_{i=1}^{p} \partial f_{i}(\bar{x}) \\
\langle\zeta, z\rangle \leq 0 \text { for all } \zeta \in \partial g_{t}(\bar{x}), t \in \hat{T}(\bar{x})
\end{array}\right.
$$

has no solution $z \in \mathbb{R}^{n}$.

Proof Suppose to the contrary that (3.1) has a solution $z$. Then $z \neq 0$ and $z \in C(\bar{x})$. Without loss of generality, we can assume that

$$
\begin{aligned}
& \langle\xi, z\rangle<0 \quad \text { for all } \xi \in \partial f_{1}(\bar{x}), \\
& \langle\xi, z\rangle \leq 0 \quad \text { for all } \xi \in \bigcup_{i=2}^{p} \partial f_{i}(\bar{x}) .
\end{aligned}
$$

By our generalized Guignard constraint qualification assumption, $z \in \operatorname{cl}\left[\operatorname{co}\left(T\left(Q^{1} ; \bar{x}\right)\right)\right]$, and hence there exists a sequence $\left\{z^{m}\right\}_{m=1}^{\infty} \subset \operatorname{co}\left(T\left(Q^{1} ; \bar{x}\right)\right)$ such that

$$
\lim _{m \rightarrow \infty} z^{m}=z
$$

For each $z^{m}, m=1,2, \ldots$, there exist numbers $L_{m}$ and $\lambda_{m l} \geq 0$, and $z^{m l} \in T\left(\Omega^{1} ; \bar{x}\right), z^{m l} \neq 0$, $l=1,2, \ldots, L_{m}$, such that

$$
\sum_{l=1}^{L_{m}} \lambda_{m l}=1, \quad \sum_{l=1}^{L_{m}} \lambda_{m l} z^{m l}=z^{m} .
$$


Since, for each $m=1,2, \ldots$ and $l=1,2, \ldots, L_{m}, z^{m l} \in T\left(Q^{1} ; \bar{x}\right)$, there exist sequences $\left\{x^{m l n}\right\}_{n=1}^{\infty} \subset Q^{1}$ and $\left\{t_{m l n}\right\}_{n=1}^{\infty} \subset \mathbb{R}$, with $t_{m l n}>0$ for all $n$, such that

$$
\lim _{n \rightarrow \infty} x^{m l n}=\bar{x}, \quad \lim _{n \rightarrow \infty} t_{m l n}\left(x^{m l n}-\bar{x}\right)=z^{m l} .
$$

Noticing that $x^{m l n} \in Q^{1}$ for all $n$, we have $x^{m l n} \in \Omega$ and $f_{i}\left(x^{m l n}\right) \leq f_{i}(\bar{x})+c_{i}\left\|x^{m l n}-\bar{x}\right\|^{m}$ for $i=2,3, \ldots, p$.

Since $\bar{x}$ is a semi-strict minimizer of order $m$ for (P), for all $n$ we may assume

$$
f_{1}\left(x^{m l n}\right) \geq f_{1}(\bar{x})+c_{1}\left\|x^{m l n}-\bar{x}\right\|^{m}
$$

Since $z^{m l} \neq 0$, for each $m=1,2, \ldots$ and $l=1,2, \ldots, L_{m}$, we must have $t_{m l n} \rightarrow+\infty$ as $n \rightarrow$ $+\infty$, and hence $x^{m l n}-\frac{1}{t_{m l n}} z^{m l} \rightarrow \bar{x}$ as $n \rightarrow+\infty$. Therefore,

$$
\begin{aligned}
f_{1}^{0}\left(\bar{x} ; z^{m l}\right) & =\limsup _{\substack{y \rightarrow \bar{x} \\
t \downarrow 0}} \frac{f_{1}\left(y+t z^{m l}\right)-f_{1}(y)}{t} \\
& \geq \lim _{n \rightarrow \infty} \frac{f_{1}\left(x^{m l n}-\frac{1}{t_{m l n}} z^{m l}+\frac{1}{t_{m l n}} z^{m l}\right)-f_{1}\left(x^{m l n}-\frac{1}{t_{m l n}} z^{m l}\right)}{\frac{1}{t_{m l n}}} \\
& \geq \limsup _{n \rightarrow \infty} \frac{f_{1}\left(x^{m l n}\right)-f_{1}(\bar{x})+f_{1}(\bar{x})-f_{1}\left(x^{m l n}-\frac{1}{t_{m l n}} z^{m l}\right)}{\frac{1}{t_{m l n}}} \\
& \geq \limsup _{n \rightarrow \infty} \frac{f_{1}\left(x^{m l n}\right)-f_{1}(\bar{x})}{\frac{1}{t_{m l n}}}-\limsup _{n \rightarrow \infty} \frac{\left|f_{1}(\bar{x})-f_{1}\left(x^{m l n}-\frac{1}{t_{m l n}} z^{m l}\right)\right|}{\frac{1}{t_{m l n}}} \\
& \geq \limsup _{n \rightarrow \infty} \frac{c_{1}\left\|x^{m l n}-\bar{x}\right\|^{m}}{\frac{1}{t_{m l n}}}-\limsup _{n \rightarrow \infty} \frac{\left|f_{1}(\bar{x})-f_{1}\left(x^{m l n}-\frac{1}{t_{m l n}} z^{m l}\right)\right|}{\frac{1}{t_{m l n}}}
\end{aligned}
$$

(by (3.5))

$$
\begin{aligned}
& \geq-\lim \sup _{n \rightarrow \infty} \frac{\left|f_{1}(\bar{x})-f_{1}\left(x^{m l n}-\frac{1}{t_{m l n}} z^{m l}\right)\right|}{\frac{1}{t_{m l n}}} \\
& \geq-\lim \sup _{n \rightarrow \infty} t_{m l n} K_{1}\left|\bar{x}-x^{m l n}+\frac{1}{t_{m l n}} z^{m l}\right|
\end{aligned}
$$

(by the Lipschitz continuity of $f_{1}$ )

$$
\begin{aligned}
& =-\lim \sup _{n \rightarrow \infty} K_{1}\left|t_{m l n}\left(\bar{x}-x^{m l n}\right)+z^{m l}\right| \\
& =0 \quad(\text { by (3.4)). }
\end{aligned}
$$

Because $f_{1}(x)$ is strictly differentiable at $\bar{x}$, we know $f_{1}^{0}(x ; v)=\left\langle D_{s} f_{1}(\bar{x}), v\right\rangle$. By Proposition 2.2.4 in [18], we also know that $\partial f_{1}(\bar{x})=\left\{D_{s} f_{1}(\bar{x})\right\}$. In view of (3.3) we obtain $\left\langle D_{s} f_{1}(\bar{x}), z^{m}\right\rangle=f_{1}^{0}\left(\bar{x} ; z^{m}\right) \geq 0$, which further gives us $\left\langle D_{s} f_{1}(\bar{x}), z\right\rangle=f_{1}^{0}(\bar{x} ; z) \geq 0$ because of (3.2), contradicting the assumption that $z$ is a solution of the system (3.1). Therefore, under the assumption that the generalized Guignard constraint qualification holds and $f_{i}(x)$, $i \in P$, are strictly differentiable at $\bar{x}$, (3.1) has no solution $z \in \mathbb{R}^{n}$.

Now let us show that (3.1) has no solution $z \in \mathbb{R}^{n}$ under the generalized Abadie constraint qualification. Suppose to the contrary that (3.1) has a solution $z$. Then $z \neq 0$ and 
$z \in C(\bar{x})$. By the generalized Abadie constraint qualification, without loss of generality we may assume that $z \in T\left(Q^{1} ; \bar{x}\right)$, and hence there exist sequences $\left\{x^{n}\right\}_{n=1}^{\infty} \subset Q^{1}$ and $\left\{t_{n}\right\}_{n=1}^{\infty} \subset \mathbb{R}$, with $t_{n}>0$ for all $n$, such that

$$
\lim _{n \rightarrow \infty} x^{n}=\bar{x}, \quad \lim _{n \rightarrow \infty} t_{n}\left(x^{n}-\bar{x}\right)=z .
$$

Replacing $t_{m l n}$ by $t_{n}, x^{m l n}$ by $x^{n}$, and $z^{m l}$ by $z$ in (3.6), we arrive at $f_{1}^{0}(\bar{x} ; z) \geq 0$. By Proposition 2.1.2 in [18], we know that there is a $\xi \in \partial f_{1}(\bar{x})$ such that $\langle\xi, z\rangle=f_{1}^{0}(\bar{x} ; z) \geq 0$, contradicting the assumption that $z$ is a solution of the system (3.1). Therefore, (3.1) has no solution $z \in \mathbb{R}^{n}$.

Now we are ready to prove the following necessary optimality condition for (P).

Theorem 3.1 Let $\bar{x} \in \Omega$ and let the functions $f_{i}(x)$ for $i \in P$ and $g_{t}(x)$ for $t \in T$ be Lipschitz at $\bar{x}$. If $\bar{x}$ is a semi-strict minimizer of order $m$ for $(\mathrm{P})$, if the generalized Guignard constraint qualification holds at $\bar{x}$ and $f_{i}(x)$ for each $i \in P$ is strictly differentiable at $\bar{x}$ (or the generalized Abadie constraint qualification holds at $\bar{x}$ ), and if for each $i_{0} \in P$, the set cone $\left(\left\{\zeta \in \partial g_{t}(\bar{x}): t \in \hat{T}(\bar{x})\right\} \cup\left\{\xi \in \partial f_{i}(\bar{x}): i \in P, i \neq i_{0}\right\}\right)$ is closed, then there exist $u^{*} \in U \equiv\left\{u \in \mathbb{R}^{p}: u>0, \sum_{i=1}^{p} u_{i}=1\right\}$, integers $v^{*}$, with $0 \leq v^{*} \leq n+1$, such that there exist $t^{m} \in \hat{T}(\bar{x})$ and $v_{m}^{*}>0, m \in\left\{1,2, \ldots, v^{*}\right\}$, with the property that

$$
0 \in \sum_{i=1}^{p} u_{i}^{*} \partial f_{i}(\bar{x})+\sum_{m=1}^{\nu^{*}} v_{m}^{*} \partial g_{t^{m}}(\bar{x}) .
$$

Proof In Lemma 3.2, set

$$
\begin{aligned}
& A^{i}=\partial f_{i}(\bar{x}), \quad i \in P, \\
& B=\bigcup_{t \in \hat{T}(\bar{x})} \partial g_{t}(\bar{x}) .
\end{aligned}
$$

By Proposition 2.1.2 in [18], we know that $A^{i}$ is a compact convex set. According to Lemma 3.3, the system (3.1) has no solution and, therefore, by Lemma 3.2, there exist $u^{*} \in U, a^{i} \in \partial f_{i}(x)$ for $i \in P$, integers $v^{*}$ with $0 \leq v^{*} \leq n+1$, such that there exist $v^{*}$ points $t^{m} \in \hat{T}(\bar{x})$ and $v^{*}$ positive numbers $v_{m}^{*}>0, m \in\left\{1,2, \ldots, v^{*}\right\}$, such that (3.7) holds.

Remark 3.1 If we modify the definition of $Q^{i}(\bar{x})$ as follows:

$$
Q^{i}(\bar{x}):=\left\{x \in \mathbb{R}^{n}: f_{k}(x)<f_{k}(\bar{x})+c_{k}\|x-\bar{x}\|, k \in P \text { and } k \neq i\right\} \cap \Omega,
$$

and define the generalized Guignard constraint qualification and generalized Abadie constraint qualification accordingly, we can prove a similar necessary result for $\bar{x}$ to be a strict minimizer of order $m$ for $(\mathrm{P})$.

\section{Sufficient conditions}

In this section we discuss sufficient optimality results under various generalized higherorder strong convexity (introduced in Section 2) hypotheses imposed on the involved functions. 
Theorem 4.1 (Sufficient optimality conditions I) Let $\bar{x} \in \Omega$ and $\hat{T}(\bar{x}) \neq \emptyset$. Suppose that there exist scalars $\alpha_{i} \geq 0, i=1,2, \ldots, p$ with $\sum_{i=1}^{p} \alpha_{i}=1$, and $\beta_{t} \geq 0, t \in \hat{T}(\bar{x})$ with $\beta_{t} \neq 0$ for finitely many indices $t$, such that

$$
0 \in \sum_{i=1}^{p} \alpha_{i} \partial f_{i}(\bar{x})+\sum_{t \in \hat{T}(\bar{x})} \beta_{t} \partial g_{t}(\bar{x}) .
$$

If the functions $f_{i}, i=1,2, \ldots, p$, are strongly convex of order $m$ at $\bar{x}$, and $g_{t}$ for $t \in \hat{T}(\bar{x})$ and $\beta_{t} \neq 0$, are strongly quasiconvex of order $m$ at $\bar{x}$, then $\bar{x}$ is a strict minimizer of order $m$ for $(\mathrm{P})$.

Proof Let $J(\bar{x}):=\left\{t \in \hat{T}(\bar{x}): \beta_{t} \neq 0\right\}$. Because of (4.1), we derive that there exist $\xi_{i} \in \partial f_{i}(\bar{x})$ for $i \in\{1,2, \ldots, p\}$ and $\zeta_{t} \in \partial g_{t}(\bar{x})$ for $t \in J(\bar{x})$ such that

$$
\sum_{i=1}^{p} \alpha_{i} \xi_{i}+\sum_{t \in J(\bar{x})} \beta_{t} \zeta_{t}=0
$$

Since $f_{i}$ for $i \in\{1,2, \ldots, p\}$ are strongly convex of order $m$ at $\bar{x}$, we see that there exist $\bar{c}_{i}>0$ for $i \in\{1,2, \ldots, p\}$ such that

$$
f_{i}(x)-f_{i}(\bar{x}) \geq\left\langle\xi_{i}, x-\bar{x}\right\rangle+\bar{c}_{i}\|x-\bar{x}\|^{m} \quad \text { for all } x \in \Omega, \xi_{i} \in \partial f_{i}(\bar{x}), i=1,2, \ldots, p \text {. }
$$

Noticing $\alpha_{i} \geq 0$ for $i \in\{1,2, \ldots, p\}$, we have

$$
\sum_{i=1}^{p} \alpha_{i}\left(f_{i}(x)-f_{i}(\bar{x})\right) \geq\left\langle\sum_{i=1}^{p} \alpha_{i} \xi_{i}, x-\bar{x}\right\rangle+\sum_{i=1}^{p} \alpha_{i} \bar{c}_{i}\|x-\bar{x}\|^{m} .
$$

On the other hand, $g_{t}(x) \leq g_{t}(\bar{x})=0$ for $x \in \Omega, t \in J(\bar{x})$. By the strong quasiconvexity of order $m$ at $\bar{x}$ for $g_{t}$ with $t \in J(\bar{x})$, we see that there exist $\bar{c}_{t}>0$ for $t \in J(\bar{x})$ such that, for $\zeta_{t} \in \partial g_{t}(\bar{x})$

$$
\left\langle\zeta_{t}, x-\bar{x}\right\rangle+\bar{c}_{t}\|x-\bar{x}\|^{m} \leq 0
$$

furthermore, it follows from $\beta_{t} \geq 0$ for $t \in J(\bar{x})$ that

$$
\left\langle\sum_{t \in J(\bar{x})} \beta_{t} \zeta_{t}, x-\bar{x}\right\rangle+\sum_{t \in J(\bar{x})} \beta_{t} \bar{c}_{t}\|x-\bar{x}\|^{m} \leq 0
$$

Adding (4.3) to (4.4), we get

$$
\begin{aligned}
& \sum_{i=1}^{p} \alpha_{i}\left(f_{i}(x)-f_{i}(\bar{x})\right) \\
& \quad \geq\left\langle\sum_{i=1}^{p} \alpha_{i} \xi_{i}+\sum_{t \in J(\bar{x})} \beta_{t} \xi_{t}, x-\bar{x}\right\rangle+\left(\sum_{i=1}^{p} \alpha_{i} \bar{c}_{i}+\sum_{t \in J(\bar{x})} \beta_{t} \bar{c}_{t}\right) \cdot\|x-\bar{x}\|^{m} .
\end{aligned}
$$


It follows from (4.2) that

$$
\sum_{i=1}^{p} \alpha_{i}\left(f_{i}(x)-f_{i}(\bar{x})\right)-\left(\sum_{i=1}^{p} \alpha_{i} \bar{c}_{i}+\sum_{t \in J(\bar{x})} \beta_{t} \bar{c}_{t}\right) \cdot\|x-\bar{x}\|^{m} \geq 0 .
$$

Let $\bar{c}=\sum_{i=1}^{p} \alpha_{i} \bar{c}_{i}+\sum_{t \in J(\bar{x})} \beta_{t} \bar{c}_{t}$ and $c_{i}=\alpha_{i} \bar{c}$. Noticing that $\sum_{i=1}^{p} \alpha_{i}=1$, we obtain

$$
\sum_{i=1}^{p} \alpha_{i}\left(f_{i}(x)-f_{i}(\bar{x})\right) \geq \sum_{i=1}^{p} \alpha_{i} c\|x-\bar{x}\|^{m} .
$$

This implies

$$
\left\langle\alpha, f(x)-f(\bar{x})-c\|x-\bar{x}\|^{m}\right\rangle \geq 0,
$$

where $c=(\bar{c}, \bar{c}, \ldots, \bar{c})$ with $\bar{c}>0$. Since $\alpha_{i} \geq 0$ and $\sum_{i=1}^{p} \alpha_{i}=1$, we further see that for all $x \in \Omega$

$$
f(x) \nless f(\bar{x})+c\|x-\bar{x}\|^{m},
$$

which implies that $\bar{x}$ is a strict minimizer of order $m$ for $(\mathrm{P})$.

Theorem 4.2 (Sufficient optimality conditions II) Let $\bar{x} \in \Omega$ and $\hat{T}(\bar{x}) \neq \emptyset$. Suppose that there exist scalars $\alpha_{i} \geq 0, i=1,2, \ldots, p$ with $\sum_{i=1}^{p} \alpha_{i}=1$, and $\beta_{t} \geq 0, t \in \hat{T}(\bar{x})$ with $\beta_{t} \neq 0$ for finitely many indices $t$, such that

$$
0 \in \sum_{i=1}^{p} \alpha_{i} \partial f_{i}(\bar{x})+\sum_{t \in \hat{T}(\bar{x})} \beta_{t} \partial g_{t}(\bar{x})
$$

If the functions $f_{i}, i \in\left\{1,2, \ldots, p: \alpha_{i} \neq 0\right\}$, are strongly convex of order $m$ at $\bar{x}$ and at least one of them is strictly strong convex of order $m$ at $\bar{x}$, and $g_{t}$ for $t \in \hat{T}(\bar{x})$ and $\beta_{t} \neq 0$, are strongly quasiconvex of order $m$ at $\bar{x}$, then $\bar{x}$ is a semi-strict minimizer of order $m$ for $(\mathrm{P})$.

Proof In the proof of Theorem 4.1, we derived that there exist $\xi_{i} \in \partial f_{i}(\bar{x})$ for $i \in\{1,2, \ldots, p\}$ and $\zeta_{t} \in \partial g_{t}(\bar{x})$ for $t \in J(\bar{x})$, such that

$$
\sum_{i=1}^{p} \alpha_{i} \xi_{i}+\sum_{t \in J(\bar{x})} \beta_{t} \zeta_{t}=0
$$

and that there exist $\overline{c_{t}}>0$ for $t \in J(\bar{x})$, such that, for $\zeta_{t} \in \partial g_{t}(\bar{x})$, we have

$$
\left\langle\sum_{t \in J(\bar{x})} \beta_{t} \zeta_{t}, x-\bar{x}\right\rangle+\sum_{t \in J(\bar{x})} \beta_{t} \bar{c}_{t}\|x-\bar{x}\|^{m} \leq 0
$$

Since the functions $f_{i}$ for $i \in\{1,2, \ldots, p\}$ are strongly convex of order $m$ at $\bar{x}$ and there is at least one $i_{0} \in\left\{1,2, \ldots, p: \alpha_{i} \neq 0\right\}$ such that $f_{i_{0}}$ is strictly strong convex of order $m$ at $\bar{x}$, 
using the same argument as in the proof of Theorem 4.1, we arrive at the conclusion that there exist $\bar{c}_{i}>0$ for $i \in\{1,2, \ldots, p\}$ such that

$$
\sum_{i=1}^{p} \alpha_{i}\left(f_{i}(x)-f_{i}(\bar{x})\right)>\left\langle\sum_{i=1}^{p} \alpha_{i} \xi_{i}, x-\bar{x}\right\rangle+\sum_{i=1}^{p} \alpha_{i} \bar{c}_{i}\|x-\bar{x}\|^{m} .
$$

Adding the above inequality to (4.7), we obtain

$$
\begin{aligned}
& \sum_{i=1}^{p} \alpha_{i}\left(f_{i}(x)-f_{i}(\bar{x})\right) \\
& \quad>\left\langle\sum_{i=1}^{p} \alpha_{i} \xi_{i}+\sum_{t \in J(\bar{x})} \beta_{t} \zeta_{t}, x-\bar{x}\right\rangle+\left(\sum_{i=1}^{p} \alpha_{i} \bar{c}_{i}+\sum_{t \in J(\bar{x})} \beta_{t} \bar{c}_{t}\right)\|x-\bar{x}\|^{m} .
\end{aligned}
$$

It follows from (4.6) that

$$
\sum_{i=1}^{p} \alpha_{i}\left(f_{i}(x)-f_{i}(\bar{x})\right)-\left(\sum_{i=1}^{p} \alpha_{i} \bar{c}_{i}+\sum_{t \in J(\bar{x})} \beta_{t} \overline{c_{t}}\right)\|x-\bar{x}\|^{m}>0 .
$$

Let $\bar{c}=\sum_{i=1}^{p} \alpha_{i} \bar{c}_{i}+\sum_{t \in J(\bar{x})} \beta_{t} \bar{c}_{t}$ and $c_{i}=\alpha_{i} \bar{c}$. Noticing that $\sum_{i=1}^{p} \alpha_{i}=1$, we get

$$
\sum_{i=1}^{p} \alpha_{i}\left(f_{i}(x)-f_{i}(\bar{x})\right)>\sum_{i=1}^{p} \alpha_{i} c\|x-\bar{x}\|^{m} .
$$

Hence, with $c=(\bar{c}, \bar{c}, \ldots, \bar{c})$ we have

$$
\left\langle\alpha, f(x)-f(\bar{x})-c\|x-\bar{x}\|^{m}\right\rangle>0 .
$$

Because $\alpha_{i} \geq 0$ for $i \in\{1,2, \ldots, p\}$ and $\sum_{i=1}^{p} \alpha_{i}=1$, we know that, for all $x \in \Omega$,

$$
f(x) \not \leq f(\bar{x})+c\|x-\bar{x}\|^{m},
$$

which implies that $\bar{x}$ is a semi-strict minimizer of order $m$ for $(\mathrm{P})$.

Theorem 4.3 (Sufficient optimality conditions III) Let $\bar{x} \in \Omega$ and $\hat{T}(\bar{x}) \neq \emptyset$. Suppose that there exist scalars $\alpha_{i} \geq 0, i=1,2, \ldots, p$ with $\sum_{i=1}^{p} \alpha_{i}=1$, and $\beta_{t} \geq 0, t \in \hat{T}(\bar{x})$ with $\beta_{t} \neq 0$ for finitely many indices $t$, such that

$$
0 \in \sum_{i=1}^{p} \alpha_{i} \partial f_{i}(\bar{x})+\sum_{t \in \hat{T}(\bar{x})} \beta_{t} \partial g_{t}(\bar{x}) .
$$

If the functions $f_{i}, i=1,2, \ldots, p$, are strongly convex of order $m$ at $\bar{x}$, and $\sum_{t \in \hat{T}(\bar{x})} \beta_{t} g_{t}$ is strictly strong quasiconvex of order $m$ at $\bar{x}$, then $\bar{x}$ is a semi-strict minimizer of order $m$ for $(\mathrm{P})$.

Proof The proof is similar to that of Theorems 4.1 and 4.2. 


\section{Conclusions}

We have defined a strict minimizer of higher-order and a semi-strict minimizer of higherorder for a semi-infinite multi-objective optimization problem in this paper. We have presented a non-smooth semi-infinite version of the generealized Guignard and Abadie constraint qualifications. Under those constraint qualifications, utilizing the method in [15, 16] we have proved necessary optimality conditions for a semi-strict minimizer of higherorder and a strict minimizer of higher-order. Three sufficient optimality conditions have been proved under the assumption of strong convexities.

Competing interests

The author declares that he has no competing interests.

\section{Acknowledgements}

This research was supported by Natural Science Foundation of China under Grant No. 11361001; Natural Science Foundation of Ningxia under Grant No. NZ14101.

Received: 27 August 2016 Accepted: 13 October 2016 Published online: 24 October 2016

\section{References}

1. Chuong, TD: Lower semicontinuity of the Pareto solution in quasiconvex semi-infinite vector optimization. J. Math. Anal. Appl. 388, 443-450 (2012)

2. Chuong, TD, Huy, NQ, Yao, JC: Stability of semi-infinite vector optimization problems under functional perturbations. J. Glob. Optim. 45, 583-595 (2009)

3. Chuong, TD, Huy, NQ, Yao, JC: Pseudo-Lipschitz property of linear semi-infinite vector optimization problems. Eur. J. Oper. Res. 200, 639-644 (2010)

4. Chuong, TD, Yao, JC: Sufficient conditions for pseudo-Lipschitz property in convex semi-infinite vector optimization problems. Nonlinear Anal. 71, 6312-6322 (2009)

5. Huy, NQ, Kim, DS: Lipschitz behavior of solutions to nonconvex semi-infinite vector optimization problems. J. Glob. Optim. 56, 431-448 (2013)

6. Fan, $\mathrm{X}$, Cheng, C, Wang, H: Density of stable convex semi-infinite vector optimization problems. Oper. Res. Lett. 40, 140-143 (2012)

7. Caristi, G, Ferrara, M, Stefanescu, A: Semi-infinite multiobjective programming with generalized invexity. Math. Rep. $12,217-233$ (2010)

8. Kanzi, N, Nobakhtian, S: Optimality conditions for nonsmooth semi-infinite multiobjective programming. Optim. Lett. 8, 1517-1528 (2014)

9. Chuong, TD, Kim, DS: Nonsmooth semi-infinite multiobjective optimization problems. J. Optim. Theory Appl. 160 748-762 (2014)

10. Jiménez, B: Strict efficiency in vector optimization. J. Math. Anal. Appl. 265, 264-284 (2002)

11. Auslender, A: Stability in mathematical programming with non-differentiable data. SIAM J. Control Optim. 22, 239-254 (1984)

12. Ward, DE: Characterizations of strict local minima and necessary conditions for weak sharp minima. J. Optim. Theory Appl. 80, 551-571 (1994)

13. Bhatia, G: Optimality and mixed saddle point criteria in multiobjective optimization. J. Math. Anal. Appl. 342, 135-145 (2008)

14. Lin, GH, Fukushima, M: Some exact penalty results for nonlinear programs and mathematical programs with equilibrium constraints. J. Optim. Theory Appl. 118,67-80 (2003)

15. Maeda, T: Constraint qualifications in multiobjective optimization problems: differentiable case. J. Optim. Theory Appl. 80, 483-500 (1994)

16. Preda, V, Chitescu, I: On constraint qualification in multiobjective optimization problems: semidifferentiable case. J. Optim. Theory Appl. 100, 417-433 (1999)

17. Zalmai, GJ, Zhang, QH: Global semiparametric sufficient efficiency conditions for semiinfinite multiobjective fractional programming problems containing generalized $(\alpha, \eta, \rho)$-V-invex functions. Southeast Asian Bull. Math. 32 , 573-599 (2008)

18. Clarke, FH: Optimization and Nonsmooth Analysis. Wiley, New York (1983)

19. Clarke, FH, Ledyaev, YS, Stern, RJ, Wolenski, PR: Nonsmooth Analysis and Control Theory. Springer, New York (1998)

20. Goberna, MA, López, MA: Linear Semi-Infinite Optimization. Wiley, New York (1998) 